\title{
Isolated renal mucormycosis
}

\author{
H.D. Flood ${ }^{1}$, A.M. O'Brien² and D.G. Kelly ${ }^{1}$ \\ ${ }^{1}$ Department of Urology; ${ }^{2}$ Department of Pathology, St Vincent's Hospital, Dublin 4, Republic of Ireland.
}

\begin{abstract}
Summary: Isolated renal mucormycosis is very rare and we describe such a case masquerading as a hypernephroma in a poorly-controlled diabetic.
\end{abstract}

\section{Introduction}

Isolated renal involvement is an unusual manifestation of mucormycosis. To our knowledge only two previous cases have been reported.

\section{Case report}

A 68 year old male, maturity-onset diabetic presented with a 12 month history of intermittent left loin pain and a single episode of frank haematuria. His diabetes, diagnosed 11 years previously, was poorly controlled on oral hypoglycaemic agents. On physical examination the only relevant abnormal finding was left loin tenderness. Initial investigations showed haemoglobin concentration $11.2 \mathrm{~g} / \mathrm{dl}$, white cell count was normal, erythrocyte sedementation rate was $90 \mathrm{~mm}$ in the first hour. Blood sugar varied from 1.7 to $19.3 \mathrm{mmol} / \mathrm{l}$ and glycosuria was intermittent. A mid-stream specimen of urine was blood stained and contained greater than 100 white cells $/ \mathrm{mm}^{3}$. Culture yielded no significant bacterial growth. Ziehl-Nielsen stain and cultures for tubercle bacilli were negative. Chest X-ray was normal. Intravenous urogram showed a normal right kidney and a poorly functioning left kidney with a distorted collecting system. Ultrasound examination showed areas of mixed echogenicity in the upper pole, and needle aspiration showed inflammatory cells only. Selective renal angiography showed splaying of the intrarenal vessels suggestive of an infiltrative process, such as lymphoma or multiple metastases rather than the clinically suspected diagnosis of hypernephroma. At exploration the left kidney was enlarged and studded with microabcesses and an open renal biopsy was performed. Frozen sections and subsequent paraffin sections showed necrotizing granulomatous inflammation. Ziehl-Nielsen and fungal stains were

H.D. Flood, F.R.C.S.I.; A.M. O'Brien, M.R.C.Path.; D.G. Kelly, M.Ch., F.R.C.S.I.

Accepted: 29 February 1984 negative. Routine cultures and culture for tubercle bacilli were negative. Fungal cultures were not done. Six days postoperatively a left nephrectomy was performed for persistent severe haematuria. The resected left kidney was $14 \mathrm{~cm}$ long and weighed $400 \mathrm{~g}$. Gross examination revealed widely disseminated abcesses ranging in size from 0.2 to $2 \mathrm{~cm}$ with necrosis of a number of papillae. Microscopy showed areas of suppuration and necrotizing granulomata containing fungal hyphae characteristic of mucormycosis (Chandler et al., 1980) (Figure 1).

Gram and Ziehl-Nielsen stains were negative. The histological diagnosis was of renal mucormycosis. Cultural confirmation was not obtained as unfixed tissue was not available. Post-operative blood and urine cultures for mucor were negative. The patient made an uneventful post-operative recovery and continues to do well without anti-fungal therapy.

\section{Discussion}

Mucormycosis is an uncommon fungal infection caused by certain species of Mucor, Rhizopus and Absidia. The condition characteristically occurs as an opportunistic infection in diabetics and immunocompromised individuals. A variety of clinical forms are recognised (Edwards, 1980), rhinocerebral, pulmonary, gastrointestinal, cutaneous and disseminated. Infection occurs by inhalation or ingestion and with the exception of the cutaneous form is usually acute and often fatal. Dissemination of the organism in the body is frequent and is attributable to its angioinvasive tendency. Renal involvement is seen in the disseminated form of the disease but to our knowledge only two cases of isolated renal mucormycosis have been reported. Prout and Goddard (1960) reported a case of isolated renal mucormycosis accompanied by considerable constitutional upset. Symmers (1975) reported isolated involvement of a transplanted kid- 


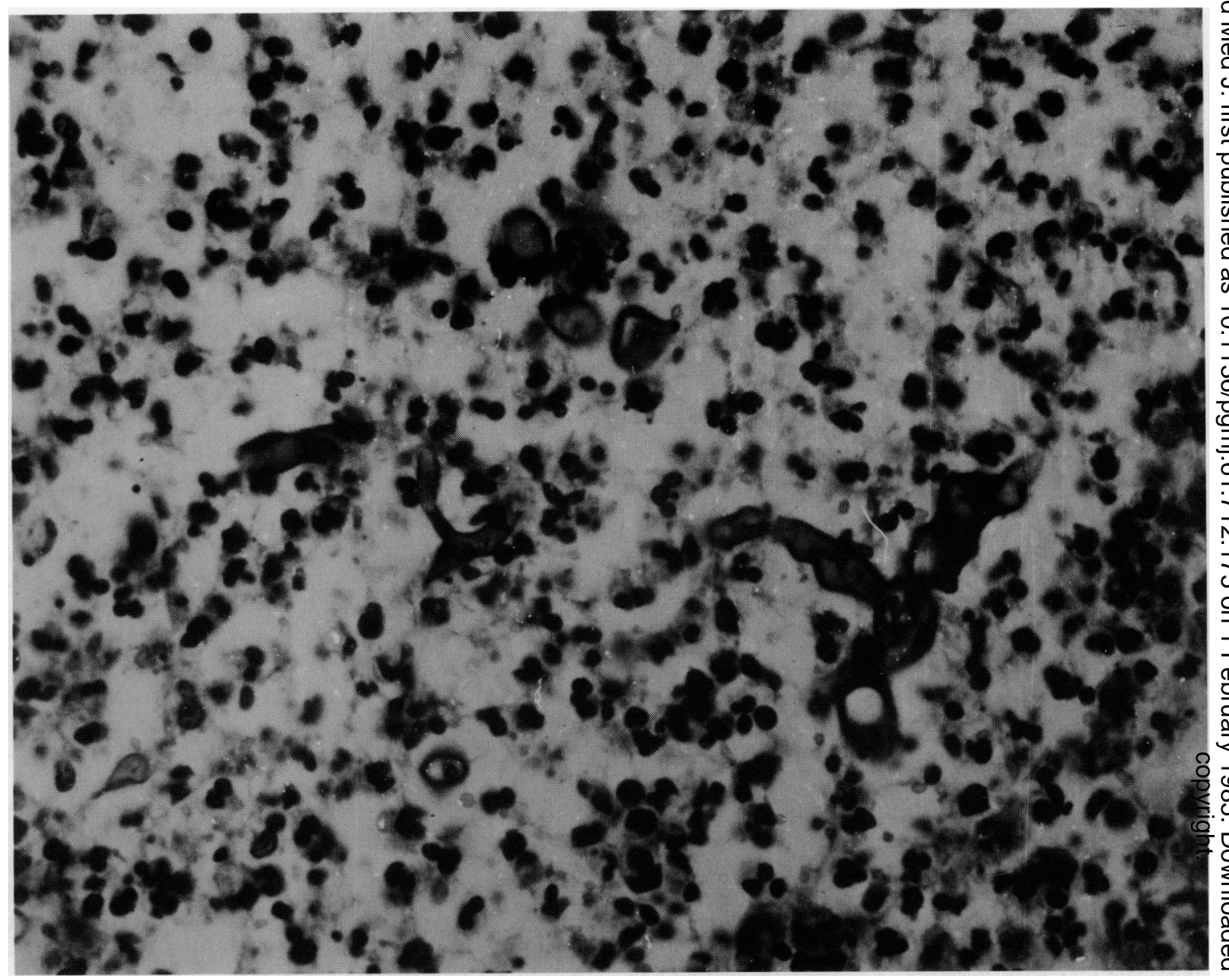

Figure 1 Photomicrograph of an area of suppuration showing broad, branched, thin-walled, non-septate hyphae of variable size and shape, with non-parallel sides and focal slightly bulbous dilatations characteristic of mucormycosis. (PAS stain X 50)

ney. Cultural confirmation was not obtained in either case. Our case is a further documentation of isolated renal mucormycosis. In our patient diabetes mellitus was the predisposing factor. In the absence of an

\section{References}

CHANDLER, F. KAPLAN, W. \& AJELlO, L. (1980). Zygomycosis. In A Colour Atlas and Textbook of the Histopathology of Mycotic Disease, p. 125. Wolfe, London.

EDWARDS, J.E. (1980). Clinical aspects of mucormycosis. In Mucormycosis Lehrer, R.I. (Moderator). Annals of Internal Medicine, 93, 96.

PROUT, G.R. \& GODDARD, R. (1960). Renal mucormycosis. New England Journal of Medicine, 263, 1246. identified primary focus we can only speculate that he sustained a sub-clinical primary infection with 3 . haematogeneous dissemination to the kidney in a manner comparable with renal tuberculosis.

SYMMERS, W. STC. (1975). Histopathology of opportunistic $\widetilde{\Omega}$ fungal infections, In Opportunistic Fungal Infections: $N$ Proceedings of the Second International Conference, Springfield Illinois. Chick, E.W., Balous, A., Furcolou, M.L. (eds), p. 276. 